\title{
Enhanced reverse saturable absorption and optical limiting in heavy-atom-substituted phthalocyanines
}

\author{
Joseph W. Perry, Kamjou Mansour, Seth R. Marder, and Kelly J. Perry \\ Jet Propulsion Laboratory, California Institute of Technology, Pasadena, California 91109 \\ Daniel Alvarez, Jr., ${ }^{*}$ and Ingrid Choong \\ Beckman Institute, California Institute of Technology, Pasadena, California 91125
}

Received February 3, 1994

\begin{abstract}
The reverse saturable absorption and the optical-limiting response of metal phthalocyanines can be enhanced by use of the heavy-atom effect. Phthalocyanines containing heavy-metal atoms, such as In, Sn, and Pb, show a nearly factor-of-2 enhancement in the ratio of effective excited-state to ground-state absorption cross sections compared with those containing lighter atoms, such as $\mathrm{Al}$ and $\mathrm{Si}$. In an $f / 8$ optical geometry, homogeneous solutions of heavy-metal phthalocyanines, at 30\% linear transmission, limit 8-ns 532-nm laser pulses to $\leq 3 \mu \mathrm{J}$ (the energy for $50 \%$ probability of eye damage) for incident energies as high as $800 \mu \mathrm{J}$.
\end{abstract}

Materials that exhibit reverse saturable absorption (RSA) are currently of interest for use in opticallimiting devices for protection of sensors and eyes from energetic light pulses. RSA can occur when states with an absorption cross section $\left(\sigma_{e}\right)$ in excess of the ground-state cross section $\left(\sigma_{g}\right)$ are produced. RSA in the visible spectrum that is due to electronic excitation was reported for several classes of organic chromophores. ${ }^{1-7}$ The effectiveness of RSA molecules for optical limiting is determined mainly by the ratio of cross sections $\left(\sigma_{e} / \sigma_{g}\right)$, which is function of wavelength, and the populations of the states that evolve in time during the pulse. There is considerable interest in approaches to enhancing the performance of RSA chromophores.

RSA and optical limiting in metallophthalocyanines (MPc's) such as chloroaluminum phthalocyanine (CAP) and bis[tri-( $n$-hexyl)siloxy] silicon naphthalocyanine ( $\mathrm{SiNc}$ ) have been investigated ${ }^{4,7}$ on picosecond and nanosecond time scales at $532 \mathrm{~nm}$. Picosecond experiments ${ }^{7}$ showed that values for $\sigma_{s} / \sigma_{g}$, where $s$ refers to the excited-singlet state, were 10.5 and 14 for CAP and SiNc, respectively. Nanosecond measurements on CAP (Ref. 8) and SiNc (Ref. 9) indicate that $\sigma_{t} / \sigma_{g}$ values ( $t$ refers to the triplet state) are $\sim 20$ to 50 . However, the triplet populations achievable during nanosecond pulses are limited by the small intersystem crossing rate, $k_{\text {isc }}$, for these molecules. . $^{9,10}$ These observations suggested to us the use of the heavy-atom effect as an approach to enhancing the optical-limiting performance of phthalocyanines. When the atomic number of the central metal atom is increased, $k_{\text {isc }}$ for the $\pi$-electronic states can be increased,,$^{10}$ permitting more efficient population of the triplet state.

In this Letter we examine the RSA performance of MPc's containing metals (and metalloids) from groups IIIA ( $\mathrm{Al}, \mathrm{Ga}, \mathrm{In}$ ) and IVA (Si, Ge, $\mathrm{Sn}$, and $\mathrm{Pb}$ ). For $\mathrm{Al}, \mathrm{Ga}$, and In phthalocyanines (Pc's) the molecular structures were of the form [tri-( $n$-hexyl)siloxy] MPc, whereas $\mathrm{Si}, \mathrm{Ge}$, and Sn Pc's were of the form bis[tri(n-hexyl)siloxy] MPc and $\mathrm{Pb} \mathrm{Pc}$ was of the form $\mathrm{Pb}$ tetra( $t$-butyl)Pc. These molecules were synthesized according to literature methods. ${ }^{11}$ The electronic absorption spectrum of $\mathrm{Sn} \mathrm{Pc}$ is shown in Fig. 1, which reveals a strong $Q$-band absorption at $678 \mathrm{~nm}$ and a region of weak absorption from $\sim 425$ to $600 \mathrm{~nm}$. Also shown is the transient triplet-triplet absorption spectrum, which shows a maximum at $510 \mathrm{~nm}$ and a bandwidth of $\sim 130 \mathrm{~nm}$. The ground-state and triplet-triplet spectra for the other molecules are quite similar, with a slight red shift of the $Q$ band as the metal becomes heavier.

The values of the triplet quantum yield $\left(\Phi_{t}\right)$ and the first excited-singlet lifetime $\left(\tau_{s}\right)$ are given in Table 1 for the group IIIA and group IVA MPc's. Indeed, the $\tau_{s}$ values decrease and the $\Phi_{t}$ values increase as

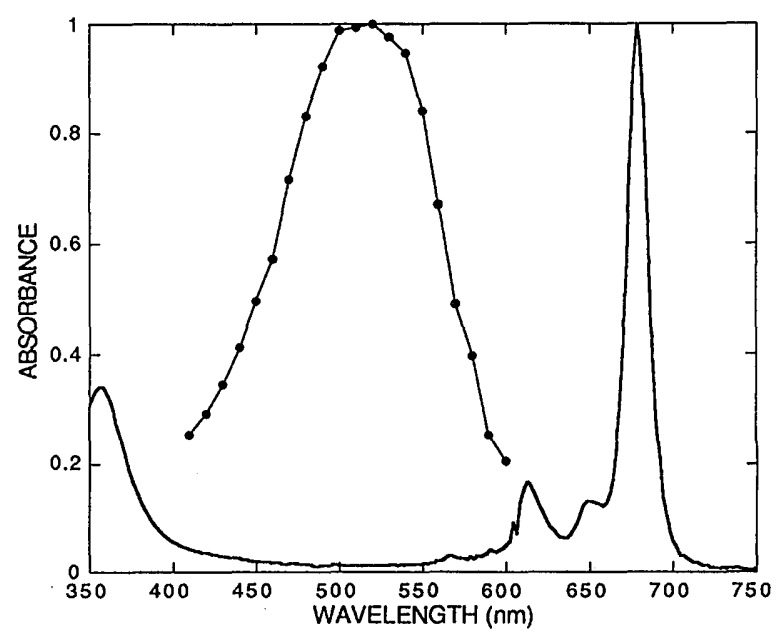

Fig. 1. Electronic absorption spectrum of bis[tri- $(n-$ hexyl)siloxy]SnPc in toluene solution at $7.1 \times 10^{-6} \mathrm{M}$. Also shown (curve with circles) is the transient absorption spectrum (arbitrary units) of Sn Pc in toluene obtained $100 \mathrm{~ns}$ after excitation at $355 \mathrm{~nm}$. 
Table 1. Photophysical Properties for Group IIIA and Group IVA Metal Pc's

\begin{tabular}{cclc}
\hline Metal & $\tau_{s}(\mathrm{~ns})^{a}$ & $\Phi_{t}{ }^{b}$ & $f_{t}(8 \mathrm{~ns})$ \\
\hline $\mathrm{Al}$ & 6.9 & 0.35 & 0.18 \\
$\mathrm{Ga}$ & 3.3 & 0.51 & 0.42 \\
$\mathrm{In}$ & 0.3 & 0.88 & 0.95 \\
$\mathrm{Si}$ & $4.5^{c}$ & $0.35^{d}$ & 0.25 \\
$\mathrm{Ge}$ & $4.2^{c}$ & $0.37^{d}$ & 0.28 \\
$\mathrm{Sn}$ & $2.0^{c}$ & 0.62 & 0.63 \\
$\mathrm{~Pb}$ & 0.35 & 0.92 & 0.95 \\
\hline
\end{tabular}

${ }^{a}$ First excited-state lifetimes determined by picosecond pump-probe measurements at $700 \mathrm{~nm}$; uncertainty is $\pm 10 \%$.

${ }^{b}$ Triplet quantum yields determined from ground-state bleaching recovery at $700 \mathrm{~nm}$; uncertainty is \pm 0.10 .

${ }^{c}$ Values determined by use of time-correlated photon counting.

${ }^{d}$ Values estimated from fluorescence quantum yields.

the metal becomes heavier. Thus the triplet population achievable during a $Q$-switched laser pulse (e.g., 8-ns) is significantly enhanced for the molecules with large $\Phi_{t}$ values. To the extent that the different molecules have similar $\sigma_{g}, \sigma_{s}$, and $\sigma_{t}$ values, one would expect trends of increased nonlinear absorption for the heavier-metal Pc's, for pulse widths between $\sim 0.5$ and $25 \mathrm{~ns}$.

Nanosecond nonlinear transmission measurements were performed with a frequency-doubled $Q$-switched Nd:YAG laser operating in a near-Gaussian transverse mode with an 8-ns (FWHM) multimode pulse envelope. The laser pulses were focused at the center of $1-\mathrm{cm}$ path-length solution cells by a $15-\mathrm{cm}$ focal-length, 2.5-cm-diameter best-form lens ( $f / 40$ geometry) giving a waist spot size of $\sim 13 \mu \mathrm{m}$ (half-width at $1 / e^{2}$ maximum intensity) and a cellentrance spot size of $\sim 63 \mu \mathrm{m}$. The transmitted energy was collected by a $2.5-\mathrm{cm}$-diameter, $15-\mathrm{cm}$ focal-length lens located so as to relay the collected energy to a nominal spot size of $\sim 2.0 \mathrm{~mm}$ on a photodiode of $1-\mathrm{cm}$ diameter. This geometry afforded essentially total integrated energy detection, as was verified by the following experiment. Measurements with a 50\%-transmitting aperture stop in front of the detector performed on SiNc in a series of solvents with varying thermal refractive-index coefficients (i.e., nitrobenzene to carbon disulfide) showed different transmission responses; whereas, without the aperture, the responses were the same, thus demonstrating the insensitivity of the unapertured detection to refractive spot-size changes for pulse energies as high as the highest values used.

Nonlinear transmission data obtained with 532-nm, 8-ns laser pulses for the two series of MPc's are shown in Fig. 2. At incident fluences below $\sim 2 \mathrm{~mJ} / \mathrm{cm}^{2}$ the transmittance coincides with that measured by use of a spectrophotometer. The nanosecond pulse data for the two series of molecules show the trend of increased nonlinear absorption as the metal becomes heavier, consistent with an enhanced triplet contribution. Measurements on the group IVA MPc's were also performed with 70-ps pulses, which are short compared with the time scales for triplet formation in these molecules. The $\mathrm{Si}, \mathrm{Ge}$, and Sn Pc's showed the opposite trend of

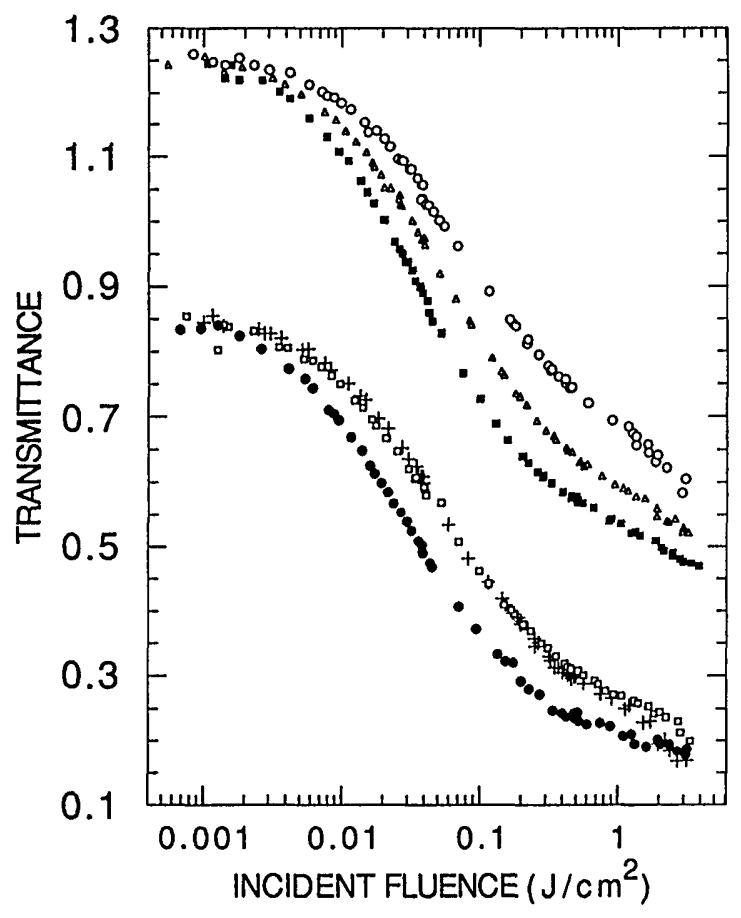

Fig. 2. Nonlinear transmittance of group IIIA (upper curves: $\mathrm{Al}$, open circles; $\mathrm{Ga}$, open triangles; In, filled squares) and group IVA (lower curves: $\mathrm{Si}$, plus sign; $\mathrm{Ge}$, open squares; Sn, filled circles) metal-substituted Pc's in toluene solution measured with 8-ns, 532-nm laser pulses and an $f / 40$ optical geometry. Solutions had $84 \%$ linear transmission at $532 \mathrm{~nm}$ for a 1-cm path length, which corresponds to a concentration of $\sim 1.4 \times 10^{-4} \mathrm{M}$. The upper curves have been displaced vertically by 0.4 for presentation.

the nonlinear transmittance compared with that for the nanosecond pulses. Since the $\sigma_{g}$ values are all $\sim 2.3 \times 10^{-18} \mathrm{~cm}^{2}$ for these molecules the picosecond results show that the relative strength of the excited-singlet absorption is opposite the apparent absorption observed on the nanosecond time scale. These results are consistent with enhanced triplet populations for the heavier-metal molecules during the longer pulses.

The nonlinear transmission functions for reverse saturable absorbers are expected to range from $T_{\text {lin }}=\exp \left(-\sigma_{g} N_{0} L\right)$ for low incident fluences to a saturated transmission $T_{\text {sat }}=\exp \left(-\sigma_{e} N_{0} L\right)$ for high degrees of excitation. When the pulse width is short compared with the excited-state (say, the triplet) lifetime, we can define a saturation fluence $F_{\text {sat }}=h \nu / \sigma_{g} \Phi_{t}$. At such high fluences the groundstate population is largely depleted, and the excitedstate population is distributed between the first excited-singlet and lowest-triplet states. A figure of merit for RSA molecules involving excited-singlet and triplet states can be defined as

$$
\sigma_{\text {eff }} / \sigma_{g}=\ln T_{\text {sat }} / \ln T_{\text {lin }} \approx\left(\sigma_{s} f_{s}+\sigma_{t} f_{t}\right) / \sigma_{g},
$$

where $\sigma_{\text {eff }}$ is an effective excited-state cross section and $f_{s}$ and $f_{t}$ are average fractional populations of the excited-singlet and triplet states during the pulse.

From Fig. 2 we estimate that $T_{\text {sat }} \sim 0.07$ for In Pc. For this molecule, $f_{t}$ (Table 1 ) is $\sim 0.95$, so we 


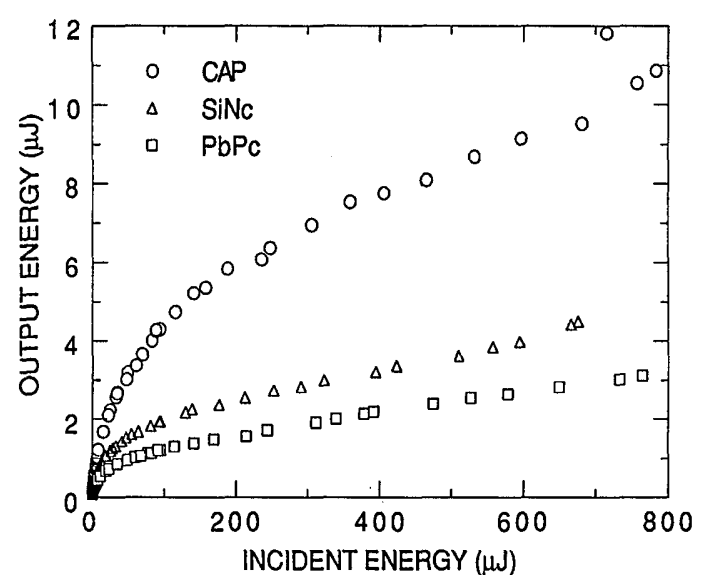

Fig. 3. The $f / 8$ optical-limiting response of $\mathrm{Pb} \mathrm{Pc}$ and SiNc in toluene and of CAP in methanol at $532 \mathrm{~nm}$. The input lens had a $2.5-\mathrm{cm}$ diameter and 4-cm focal length; the laser beam diameter was $4.8 \mathrm{~mm}$ (full width at $1 / e^{2}$ maximum intensity) at the lens; the calculated beam waist was $10 \mu \mathrm{m}$ (half-width at $1 / e^{2}$ maximum intensity); and the focus was close to the exit window of the $1-\mathrm{cm}$ cell. The transmitted energy was collected at $\sim f / 2$ and relayed to a 1.5-mm spot size on a 1-cm-diameter detector. Solutions had a linear transmittance of 0.3 at $532 \mathrm{~nm}$; concentrations were $7 \times 10^{-4} \mathrm{M}$ for SiNc, $9 \times 10^{-4} \mathrm{M}$ for $\mathrm{CAP}$, and $1.3 \times 10^{-3} \mathrm{M}$ for $\mathrm{Pb} \mathrm{Pc}$.

can ignore $f_{s}$. Using a typical $\sigma_{t}$ value of $50 \times$ $10^{-18} \mathrm{~cm}^{2}$ for MPc's, we calculate $T_{\text {sat }}=0.02$. This value is smaller than that observed, yet the fluence exiting the sample was approximately equal to $F_{\text {sat }}$ $\left(0.24 \mathrm{~J} / \mathrm{cm}^{2}\right.$ for In $\left.\mathrm{Pc}\right)$, suggesting that uniform onaxis saturation was being approached. In any case we can use the high-fluence transmittance to place a lower bound on $\sigma_{\text {eff }} / \sigma_{g}$ for the various molecules. The cross-section ratios range from 10 to 16 for the group IIIA MPc's and from 10 to 18 for the group IVA MPc's as the metal becomes heavier. In comparison, the fullerene $\mathrm{C}_{60}$ was reported ${ }^{12}$ to have a $\sigma_{t} / \sigma_{g}$ value of $\sim 3$ at $532 \mathrm{~nm}$ and a $\Phi_{t}$ value of $\geq 0.96$.

We have investigated the performance of the heavy-atom Pc's in a simple optical-limiting geometry. Figure 3 shows the optical-limiting response in an $f / 8$ geometry of $\mathrm{Pb} \mathrm{Pc}$ compared with those of CAP and SiNc. To our knowledge, SiNc had been the strongest known absorptive optical-limiting dye for nanosecond 532-nm pulses. The strong signal output of $\mathrm{Pb} \mathrm{Pc}$ is lower than that of CAP and SiNc by factors of 4 and 1.5, respectively. More importantly, the maximum allowable input energy for $3-\mu \mathrm{J}$ output energy, i.e., the energy corresponding to $50 \%$ probability for retinal damage to eyes, is higher than that for CAP and SiNc by factors of 16 and 2, respectively.
These results demonstrate the enhanced capability (i.e., nonlinear suppression of nanosecond 532-nm pulses by a factor of 81 for a limiter with a linear transmission of $30 \%$ in an $f / 8$ optical system) of the heavy-atom Pc's for optical-limiting applications. Recently strong optical limiting was also reported for a $\mathrm{Pb}$ (tetrakis-cumylphenoxy)Pc derivative. ${ }^{13}$ It should be emphasized that the optical-limiting geometries that have been used are far from optimal. Recent device modeling ${ }^{14}$ suggests that heavy-atom Pc materials in optimized optical-limiter designs that ensure uniform saturation could produce attenuations of $10^{4}$ for devices with $70 \%$ linear transmission.

This study was supported in part by the U.S. Army White Sands Missile Range through an agreement with NASA. D. Alvarez, Jr., acknowledges support from the James Irvine Foundation.

${ }^{*}$ Present address, 3-M Corporation, Minneapolis, Minnesota 55144.

\section{References}

1. C. R. Guiliano and L. D. Hess, IEEE J. Quantum Electron. QE-3, 338 (1967).

2. W. Blau, H. Byrne, W. M. Dennis, and J. M. Kelly, Opt. Commun. 56, 25 (1985).

3. R. C. Hoffman, K. A. Stetyick, R. S. Potember, and D. G. McLean, J. Opt. Soc. Am. B 6, 772 (1989).

4. D. R. Coulter, V. M. Miskowski, J. W. Perry, T. H. Wie, E. W. Van Stryland, and D. J. Hagan, Proc. Soc. Photo-Opt. Instrum. Eng. 1105, 42 (1989).

5. L. W. Tutt and S. W. McCahon, Opt. Lett. 15, 700 (1990).

6. L. W. Tutt and A. Kost, Nature (London) 356, 225 (1992).

7. T. H. Wie, D. J. Hagan, M. J. Sence, E. W. Van Stryland, J. W. Perry, and D. R. Coulter, Appl. Phys. B 54, 46 (1992).

8. T. Ohno, S. Kato, A. Yamada, and T. Tanno, J. Phys. Chem. 87, 775 (1983).

9. P. A. Firey, W. E. Ford, J. R. Sounik, M. E. Kenney, and M. A. Rodgers, J. Am. Chem. Soc. 110, 7626 (1988).

10. J. H. Brannon and D. Magde, J. Am. Chem. Soc. 102, 62 (1980).

11. J. N. Esposito, L. E. Sutton, and M. E. Kenney, Inorg. Chem. 6, 1116 (1967).

12. J. W. Arbogast, A. P. Darmanyan, C. S. Foote, Y. Rubin, F. N. Diederich, M. Alvarez, S. J. Anz, and R. L. Whetten, J. Phys. Chem. 1991, 11 (1991).

13. J. S. Shirk, R. G. S. Pong, F. J. Bartoli, and A. W. Snow, Appl. Phys. Lett 63, 1880 (1993).

14. P. A. Miles, "'Bottleneck' optical limiters-the optimal use of excited-state absorbers," Appl. Opt. (to be published). 